ORIGINAL

\title{
EVOLUCIÓN DEL CONSUMO DE FÁRMACOS ANTIPSICÓTICOS EN CASTILLA Y LEÓN (1990-2001)
}

Javier García del Pozo (1), Laura Isusi Lomas (2), Alfonso Carvajal García-Pando (1), Igor Martín Rodríguez (1), María Sáinz Gil (1), Victorina García del Pozo (1) y Alfonso Velasco Martín (1)

(1) Instituto de Farmacoepidemiología, Universidad de Valladolid, Valladolid (Spain).

(2) Dirección Técnica de Farmacia. Consejería de Sanidad y Bienestar Social. Junta de Castilla y León. Valladolid (Spain).

\section{RESUMEN}

Fundamento: A lo largo de la última década se han introducido nuevos fármacos y nuevos abordajes terapéuticos que permiten suponer cambios en la utilización de antipsicóticos en nuestro medio. E objetivo del presente estudio fue caracterizar el patrón de utilización entipsicóticos en Castilla y León para el periodo 1990-2001 as cóticos ha podido tener en el patrón de consumo de estos fármacos. Métodos: Los datos de consumo de medicamentos se obtuviero de la base de datos ECOM (Especialidades Consumo de Medicamentos) del Ministerio de Sanidad y Consumo. Esta base contiene información sobre el consumo de medicamentos dispensados con cargo a Sistema Nacional de Salud en farmacias comunitarias en todo el territorio nacional. Con el fin de estimar el consumo fuera del Sistem (a) tional Marketing Services) referidos a los años 2000 y 2001. Los datos

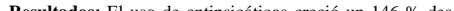

Resultados: El duso de Deipsichos crocib in $146 \%$ desc antipsicótico más utilizado en España y en Castilla y Léón. Los antipsicóticos atíícos representaron un $49 \%$ del consumo total en año 2001 y un $90 \%$ de los costes; se observa una fuerte tendencia hacia un aumento de su consumo en detrimento de los antipsicóticos típicos. Se ha estimado que un $14 \%$ de los antipsicoticos utilizados en Castilla y León lo fue fuera del Sistema Nacional de Salud. Exisen notables diferencias entre las distintas provincias.

Conclusiones: El consumo de antipsicóticos en Castilla y León creció un 146\% durante los 12 años estudiados. En este incremento, la oferta de nuevos antipsicóticos atípicos y las medidas legales relacionadas con la desinstitucionalizacios de los enfermos mentales han podido jugar un papel importante. La introducción en el merc mismos y ha ocasionado un incremento en los costes directe los consumo sin cargo al Sistema Nacional de Salud de estos medicamentos es pequeño, pero no despreciable.

Palabras clave: Agentes antipsicóticos. Farmacoepidemiologí Utilización de medicamentos. Trastornos mentales.

Correspondencia:

Dr. Alfonso Carvajal García-Pando

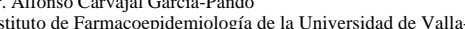
dolid

C/ Ramón y Cajal, 7. 47005 Valladolid

Correo electrónico: carvajal@ ife.uva.es

\section{ABSTRACT}

Evolution of Antipsychotic Drug

Consumption in the Autonomous

Community of Castile and Leon, Spain (1990-2001)

Background: Over the past ten years, new drugs and new approaches to treatment have been implemented making it possible ment. This study is aimed at characterizing the pattern of use of antipsychotic drugs in Castile and Leon throughout the 1990-200 period as well as ascertaining the bearing which the marketing of new antipsychotic drugs may have had on the pattern of consumption of these drugs.

Methods: The drug consumption data was obtained from the Ministry of Health and Consumer Affairs' consumption database ECOM (Especialidades Consumo de Medicamentos). This database contains information on the consumption of medications dispensed charged to the Social lnsurance system in community pharmacies Health Sy. To estimate the cons e) firm for the years 2000 and 2001 was used. The data was given in Defined Daily Doses/1000 inhabitants/day.

Results: The use of antipsychotic drugs rose by $146 \%$ within the Results: The use of antipsychotic drugs rose by 146\% within the the antipsychotic drug most used in Spain and in Castile and Leon The atypical antipsychotic drugs totaled $49 \%$ of the total consumption for 2001 and $90 \%$ of the costs, a strong trend being found toward an increase in the consumption of these atypical antipsychotic drugs in detriment to the typical antipsychotic drugs. It has been estimate that $14 \%$ of the antipsychotic drugs used in Castile and Leon wer sed outside of the National Heath Syst Cost anong he differentpovinces.

Conclusions: The consumption of antipsychotic drugs in Castile and Leon grew by $146 \%$ hroughout the twelve months studied. The related to the deinstitionlizaton of mental patients may have played a major role in this increase. The marketing of the new antipsychotic drugs has led to a change in their pattern of use and has give rise to an tise in the direct costs. The consumption of these medications no charged to the National Health System is minor, but not negligible. Key words: Antipsychotic agents. Farmacoepidemiology. Drug utilization. Mental disorders. 


\section{INTRODUCCIÓN}

Los fármacos neurolépticos o antipsicóticos constituyen el tratamiento de elección de la esquizofrenia. También se utilizan en otras indicaciones, tales como la agitación psicomotriz, los trastornos de la conducta, las demencias, los tics, el tartamudeo, el hipo rebelde y las náuseas, entre otras. Desde los años 50, cuando fueron introducidos los primeros antipsicóticos en el mercado, no se habían producido grandes cambios en este grupo farmacológico, hasta la introducción de los antipsicóticos atípicos en los años 80. Se admite, en general, que estos nuevos fármacos tienen una eficacia similar aunque presentan una menor incidencia de efectos extrapiramidales ${ }^{1}$. En cambio, su coste es muy superior al de los antipsicóticos típicos. Según datos del Sistema Nacional de Salud (SNS), durante 2001 el consumo del subgrupo N05A de la Clasificación Oficial Española de Medicamentos (neurolépticos) creció un $16,6 \%$ en importe y un $1,3 \%$ en número de envases dispensados, en total más de 224,598 millones de euros fueron gastados por el SNS en este subgrupo terapéutico, siendo el octavo subgrupo que más gasto supuso durante ese año. Olanzapina, con 99,56 millones de euros y risperidona, con 89,54 millones, respectivamente, ocuparon el quinto y décimo puesto en el gasto durante el año $2001^{2}$. Por otra parte, en estos años, el sector de seguros privados de salud ha experimentado un notable crecimiento en España, lo que hace suponer un aumento en el consumo de medicamentos en el ámbito privado $^{3}$.

En el presente trabajo se ha planteado como objetivo principal conocer cómo ha evolucionado el consumo de fármacos antipsicóticos con cargo al SNS en Castilla y León y en sus respectivas provincias, a lo largo de los últimos 12 años, comparándolo con el experimentado en España. Otros objetivos han sido conocer el impacto de la introducción de los nuevos antipsicóticos, estimar el porcentaje de consumo que para la
Comunidad Autónonoma de Castilla y León tiene lugar fuera del SNS y evaluar un posible patrón temporal en la utilización de estos fármacos.

\section{MATERIAL Y MÉTODO}

Para el presente estudio se realizó una búsqueda en la base de datos ECOM del Ministerio de Sanidad y Consumo. La misma contiene información sobre el consumo de las especialidades dispensadas en oficinas de farmacia con cargo al SNS. Se estima que el $99 \%$ de la población española está protegida por la seguridad social, por lo que esta fuente se considera habitualmente como una aproximación aceptable de la utilización real $^{4}$. Se estimaron también los costes totales de esta medicación, tanto en moneda corrientes del año considerado como en moneda constantes, descontando el efecto de la inflación. Para ello se han utilizado datos sobre el incremento del coste de la vida para el periodo considerado suministrados por el Instituto Nacional de Estadística ${ }^{5}$.

La búsqueda se realizó para el periodo 1990-2001 y para los siguientes principios activos (ATC -Anatomical Therapeutical Classification- code, N05A) ${ }^{6}$ : Fenotiazinas de cadena alifática (N05A A) -clorpromazina, levomepromazina; Fenotiazinas con estructura piperazínica (N05A B) -flufenazina, perfenazina, trifluoperazina, tioproperazina-, Fenotiazinas con estructura piperidínica (N05A C) -periciazina, tioridazina, pipotiazina-; Butirofenonas (N05A D) -haloperidol-; Derivados indólicos (N05A E) -sertindol-; Tioxantenos (N05A F) zuclopentixol-; Derivados difenilbutilpiperidina (N05A G) -pimozida-; Diazepinas, oxazepinas y tiazepinas (N05A H) -loxapina, clozapina, olanzapina, quetiapina-, Benzamidas (N05A L) -sulpirida, tiaprida-. Otros antipsicóticos (N05A X) -clotiapina, risperidona-. A su vez, y en función de sus propiedades neuroquímicas y clínicas, los antipsicóticos se clasificaron como atípicos

Rev Esp Salud Pública 2003, Vol. 77, N. ${ }^{\circ} 6$ 
(clozapina, olanzapina, risperidona, quetiapina y sertindol) o típicos (el resto). También se realizó un desglose en función de la vía de administración.

Con el fin de estimar los consumos fuera del SNS, se dispuso de los datos de ventas de antipsicóticos correspondientes a los años 2000 y 2001 para la Comunidad Autónoma de Castilla y León suministrados por IMS Health España (International Marketing Services). Este informe recoge las compras (en unidades de cada especialidad farmacéutica) de las oficinas de farmacia a los laboratorios y almacenes distribuidores. Estos datos son producidos a partir de una muestra de mayoristas que operan en todo el país, y que facilitan a IMS datos de movimiento real de unidades del mayorista a la farmacia con periodicidad mensual ó semanal. Igualmente, integra datos de una muestra de farmacias debidamente estratificada, con el fin de representar objetivamente las ventas directas laboratorio-farmacia. Se ha considerado que todas las compras de las oficinas de farmacia se traducen en ventas. La diferencia entre el consumo total y el realizado con cargo al SNS se ha considerado consumo privado. Los datos procedentes de IMS permitían un análisis por meses, lo que se ha realizado para los años 2000 y 2001.

Los datos se expresaron en dosis diarias definidas (DDD) por 1.000 habitantes y día y se utilizaron los valores de las DDD propuestos por la $\mathrm{OMS}^{6,7}$. Para los principios activos no incluidos en la clasificación ATC y para las asociaciones a dosis fijas se utilizó como DDD la dosis recomendada por el laboratorio titular de la autorización. La conversión se realizó mediante la fórmula siguiente:

número de DDD por 1.000 habitantes y día=número de envases dispensados $x$ número de formas farmacéuticas por envase x Número de mg por forma x 1.000 habitantes/ (DDD en mg x número de habitantes en la zona geográfica estudiada para el año considerado x 365 días).
Para los cálculos se han utilizado las proyecciones y estimaciones intercensales de población de hecho publicadas por el Instituto Nacional de Estadística ${ }^{8}$. No se incluyó el litio (Grupo N05A N) por no ser un neuroléptico sensu stricto y por estar indicado en el tratamiento del trastorno afectivo bipolar, ni el flupentixol, que no se encuentra comercializado como monofármaco en nuestro país sino en asociación con un antidepresivo -melitraceno-(Deanxit $($ ) $)$ y desde septiembre de 1998 se encuentra excluido de la financiación pública 9 .

\section{RESULTADOS}

Durante el periodo de 12 años que se ha considerado (1990-2001), el consumo de antipsicóticos en Castilla y León ha experimentado un crecimiento de un $146 \%$, ha pasado de 2,70 DDD/1.000 habitantes y día a 6,65 DDD/1.000 habitantes y día. Además, el consumo se ha concentrado en unos pocos principios activos; así, risperidona, olanzapina y haloperidol representaron cerca del $60 \%$ del consumo total en el año 2001. La utilización de agentes antipsicóticos típicos experimentó un notable incremento hasta el año 1996, y después un ligero descenso, mientras que la utilización de los nuevos antipsicóticos experimentó un crecimiento muy acusado desde la introducción en el mercado de risperidona y olanzapina (figura 1). Así pues, se perfila un patrón en el que aumenta notablemente el consumo de los antipsicóticos atípicos y en menor medida el de los típicos, por lo que se atisba un fenómeno de sustitución de unos por otros. En términos económicos, el crecimiento experimentado ha sido superior, de un $1245,8 \%$ en moneda corriente y de un $803,4 \%$ en moneda constante, ya que se pasó de casi 1,06 millones de euros en 1990 a más de 14,30 millones en 2001. Para el año 2001 se encontró que más del $80 \%$ del coste de todo el grupo se concentra en los antipsicóticos olanzapina y risperidona, responsables, respectivamente, del 42,7 y del $41,5 \%$ de los costes. 
Figura 1

Comparación de la evolución del consumo de antipsicóticos en Castilla y León y en España. Datos del Sistema Nacional de Salud

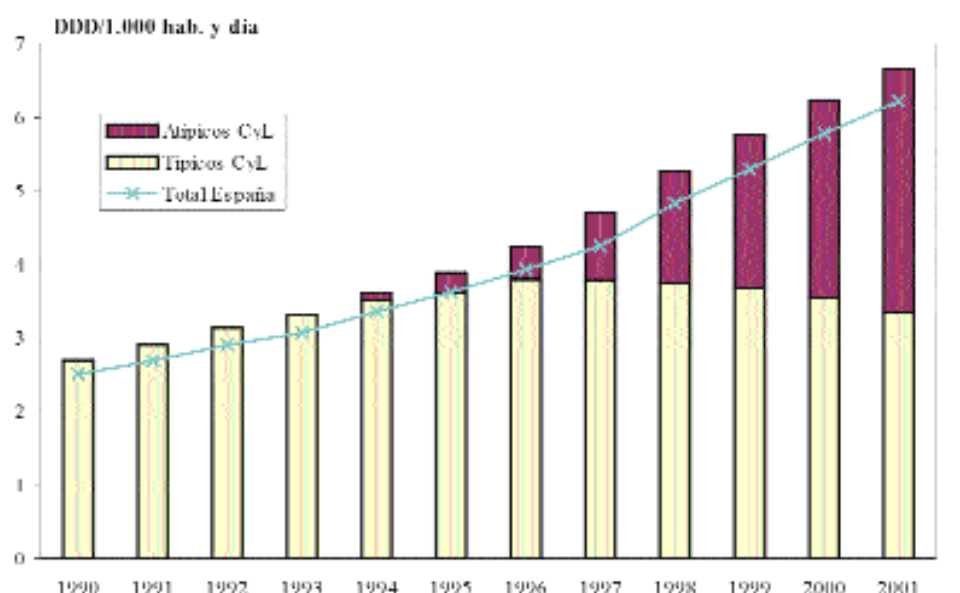

DDD utilizadas (en mg) clorpromazina oral y rectal, 300; clorpromazina parenteral 100; clotiapina oral y parenteral 80; clozapina oral 300 ; flufenazina oral 10; flufenazina parenteral 1 ; haloperidol parenteral y oral 8 ; haloperidol decanoato parenteral 3,3 ; levomepromazina oral 300 ; levomepromazina parenteral 100; loxapina parenteral y oral 100; olanzapina oral 10; perfenazina oral 30; periciazin oral 50; pimozida oral 4; pipotiazina oral 10; pipotiazina parenteral 5; quetiapina oral 400; risperidona oral 5 ; sertindol oral 16 ; sulpirida pa pis

Los porcentajes de utilización y costes de típicos y atípicos para el año 2001 se presentan en la figura 2 . El porcentaje de utilización por vía parenteral fue de un $19,9 \%$ en 1990 y de un $12,0 \%$ en 2001 ; el $99 \%$ de este consumo por vía parenteral lo fue en forma de preparados depot.

El consumo de antipsicóticos en Castilla y León es relativamente superior al de la totalidad del país. Para el periodo considerado, la utilización en Castilla y León se situó un $8,1 \%$ por encima del consumo de antipsicóticos en España. La mayor diferencia se encontró en 1997, con un consumo un 10,6\% superior en Castilla y León que en España. La menor diferencia fue para el año 2001, un $6,9 \%$ superior. Por provincias se han encontrado notables diferencias a lo largo de todo el periodo estudiado (tabla 1); así, para el 2001, Palencia y Avila, con 9,8 y 9,7 DDD por 1.000 habitantes y día fueron las provincias donde se encontró un consumo mayor, mientras que Segovia y Valladolid, con 4,2 y 4,4 DDD por 1.000 habitantes y día fueron las provincias con un menor consumo.

Utilizando los datos de IMS, se encontró que para el año 2000, el 14,7\% del consumo total de antipsicóticos en Castilla y León tuvo lugar fuera del SNS, mientras que para el 2001 este porcentaje se redujo muy ligeramente hasta el 14,1\%. Para el año 2000, la provincia con mayores diferencias entre ambas bases de datos fue Segovia, con un $20,6 \%$ de consumo fuera del SNS y la menor fue Palencia, con un 5,6\%. Finalmente, utilizando datos de IMS se calculó el consumo 
Figura 2

Porcentajes de utilización y costes de antipsicóticos en Castilla y León. Datos del Sistema Nacional de Salud. Año 2001
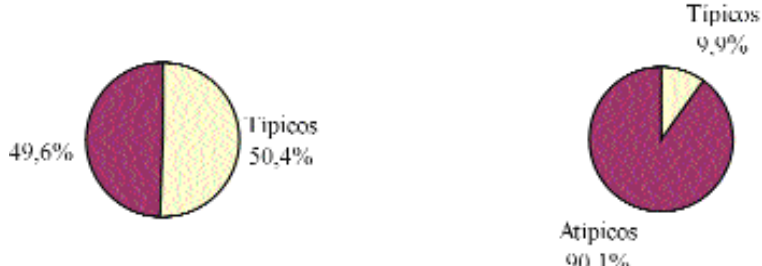

Tabla 1

Utilización de antipsicóticos en Castilla y León. Datos del Sistema Nacional de Salud expresados en DDD/1.000 habitantes y día. Período 1990-2001

\begin{tabular}{|c|c|c|c|c|c|c|c|c|c|c|c|c|}
\hline & 1990 & 1991 & 1992 & 1993 & 1994 & 1993 & 1996 & 1997 & 1998 & 1999 & 2000 & 2001 \\
\hline Nvila & 3.3 & 3.5 & 3.7 & 1.3 & 4.7 & 5.3 & 6.2 & 7.2 & 8.3 & 8.8 & 9.4 & 9.7 \\
\hline I 3urypous & 2,5 & 251 & 2,9 & 3,3 & 3,5 & 38 & $4 \pi$ & 5,5 & 6,4 & 69 & 7,1 & 7.7 \\
\hline I.xin & 2.5 & 2.6 & 2.8 & 3.1 & 3.2 & 3.5 & 3.3 & 4.1 & 1.6 & 5.2 & 5.9 & 6.5 \\
\hline Palcucia & 2.4 & 3.4 & 4.0 & 3,1 & 3.2 & 4.6 & 5.6 & 59 & 6.7 & 7,8 & 2,2 & 9.8 \\
\hline Salsmiancit & 3,3 & 3,5 & 3,8 & 4.0 & 4,3 & 4,6 & 4,9 & $.5,1$ & 5.6 & 6,1 & 6,4 & 6,9 \\
\hline Sceovia & 2.2 & 2.2 & 2.4 & 2,5 & 3.3 & 3.5 & 3.5 & 3,6 & 3,5 & 3.7 & 4,0 & 4.2 \\
\hline Simia & 4,5 & 4,9 & $5,0)$ & 5,1 & $.5,4$ & $.5,5$ & 5,3 & $.5,7$ & 6.3 & 6,7 & 7,0 & 7,5 \\
\hline Valladolid & 1.8 & 1.9 & 2.0 & 2,1 & 2.3 & 2,3 & 2.8 & 3,1 & 3.4 & 39 & 1.2 & 4.4 \\
\hline /апкма & 3,6 & 4,0 & 4,2 & 5.0 & 5,5 & 5,4 & 5,4 & 5,3 & $5 . x$ & 6,1 & 6,6 & 6,9 \\
\hline Total Cyl. & 2.7 & 2.9 & 3.1 & 3.3 & 36 & 39 & 4.2 & 4.7 & 5.3 & 5.8 & 6.2 & 6.7 \\
\hline
\end{tabular}

para los diferentes meses de los años $2000 \mathrm{y}$ 2001. En la figura 3 se presenta la evolución de este consumo.

\section{DISCUSIÓN}

El principal resultado del presente estudio ha sido el enorme crecimiento experimentado por el consumo de antipsicóticos tanto en
Castilla y León como en España. Este crecimiento se ha producido a expensas del incremento del consumo de los llamados antipsicóticos atípicos que, solo en parte, desplazan a los antipsicóticos típicos, si bien la tendencia en este sentido es hacia un progresivo desplazamiento.

La utilización de algunos agentes antipsicóticos típicos disminuyó a lo largo del 
Figura 3

Evolución del consumo de antipsicóticos en Castilla y León. Datos de IMS para los años 2000 y 2001

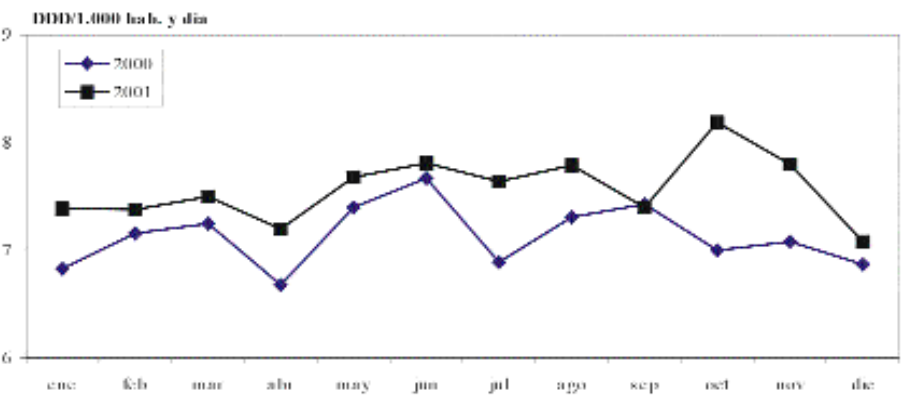

periodo estudiado, si bien para la mayoría de ellos se aprecia un crecimiento durante la primera mitad de ese periodo y una posterior disminución. Pese a ello, algunos mantienen un consumo considerable: haloperidol, zuclopentixol, flufenazina y sulpirida presentaban unos niveles de consumo altos en el 2001, siendo haloperidol el principio activo más consumido entre los antipsicóticos típicos. El crecimiento en la utilización de antipsicóticos en España ha hecho que se reduzcan las diferencias que existían con otros países a comienzos de los 90, cuando la utilización en España era notablemente más baja que en los países nórdicos ${ }^{10}$. También puede haber contribuido a esa menor diferencia el hecho de que en estos países el consumo de antipsicóticos ha disminuido en los últimos años. Entre las causas apuntadas para explicar esa disminución se encuentran una utilización de dosis más bajas, un menor número de pacientes tratados, y una disminución en la utilización de tratamientos combinados ${ }^{11}$.

El crecimiento del consumo de antipsicóticos se ha producido a expensas del crecimiento del consumo del grupo de los antipsicóticos atípicos, sobre todo de risperidona y de olanzapina; estas sustancias fueron introducidas en el mercado español en 1993 y 1997 respectivamente. En 2001 sólo estos dos principios activos constituían más del $45 \%$ del consumo total, tanto en España como en Castilla y León. El hecho de que los antipsicóticos atípicos, que constituyen el $49 \%$ del consumo, representen el $90 \%$ del coste es una invitación a la reflexión sobre el coste de estos medicamentos ${ }^{12}$. Por el contrario, clozapina, el primer antipsicótico atípico comercializado en España, no ha sido muy utilizada. Clozapina fue registrada en 1975, se retiró del mercado en 1988, y se reintrodujo en 1993 como medicamento de Especial Control Médico, sólo puede ser prescrito por especialistas en psiquiatría y los pacientes deben ser sometidos a controles hematológicos frecuentes. Además, cada prescripción dentro del SNS debe ser visada por la Inspección Médica y anotada en la historia clínica ${ }^{13}$. Todos estos factores pueden influir en su escaso consumo, pese a que clozapina es considerada como la mejor alternativa en pacientes con psicosis refractaria $^{14}$. Otro aspecto que pudiera explicar su bajo consumo es que en 1999 clozapina fue asociada con la aparición de miocarditis y cardiomiopatías fatales ${ }^{15}$. Finalmente, sertindol, un antipsicótico atípico registrado en España en 1997, fue retirado del mercado en diciembre de 1998 al asociarse con alargamiento del espacio QT en el electrocardiograma y con casos de muerte súbita. Cabe destacar, sin embargo, que en septiembre de

Rev Esp Salud Pública 2003, Vol. 77, N. ${ }^{\circ} 6$ 
2002 la Agencia Europea para la Evaluación de Medicamentos -EMEA- reevaluó positivamente este fármaco ${ }^{16}$.

Para explicar el crecimiento en el consumo de antipsicóticos como grupo debe considerarse el incremento en el número de pacientes que se tratan. A mediados de los 80 comenzó en España la llamada reforma psiquiátrica; un conjunto de medidas normativas que desarrollaban una nueva estructura de atención sanitaria para los pacientes mentales y que estipulaba la desinstitucionalización de los pacientes psiquiátricos ${ }^{17}$. Esta medida, pese a que vio la luz antes del periodo considerado en el presente estudio, tardó tiempo en ponerse en práctica, y a comienzos de los años 90 todavía quedaba por implantarse en amplias zonas de la geografía española. Los tratamientos a pacientes institucionalizados, al ser dispensados normalmente desde Servicios de Farmacia Hospitalaria, no son contabilizados por la base ECOM ni por IMS, por lo que el cambio en los procedimientos de obtención de los medicamentos para estos pacientes ha podido contribuir, al menos en parte, al incremento del consumo, ya que pacientes considerados hospitalarios pasan a considerarse ambulatorios y por tanto a obtener su medicación mediante receta médica en las oficinas de farmacia. Éste es probablemente uno de los factores que pueden explicar el crecimiento que han experimentado los preparados inyectables depot de liberación retardada. Si bien estas preparaciones facilitan el cumplimiento, disminuyendo las recidivas y hospitalizaciones y permiten mantener a los pacientes en su entorno familiar, se considera que los nuevos antipsicóticos serían mejores a este respecto ${ }^{18}$.

Otro factor que puede haber contribuido al crecimiento es el progresivo envejecimiento de la población española, lo que lleva consigo un aumento de la prevalencia de demencia en los ancianos. Aunque con los datos manejados no podemos conocer si se están utilizando los antipsicóticos para el tratamiento de las demencias seniles, es conocido que se utilizan para el manejo de este tipo de enfermos, y ello a pesar de los riesgos que presenta el empleo de estos medicamentos en ancianos (mayor predisposición a hipotensión ortostática, parkinsonismo, sedación o efectos extrapiramidales) ${ }^{19}$. Debe considerarse, a este respecto, que no todos los antipsicóticos tienen autorizada esta indicación ${ }^{20}$. Tampoco debe olvidarse una posible mayor utilización actual de estos fármacos en otras indicaciones y usos que presentan, como por ejemplo la sulpirida en el vertigo ${ }^{20}$. Otras causas que podrían explicar el crecimiento del consumo son la disponibilidad de los nuevos antipsicóticos aparentemente más seguros, el desarrollo social y económico que ha experimentado España en la última década y la progresiva implantación de unidades psiquiátricas en atención primaria y en los hospitales generales. En este sentido, no debe olvidarse que los antipsicóticos no han sido los únicos psicotropos cuyo consumo ha experimentado un notable incremento en los últimos años: los ansiolíticos e hipnóticos, y los antidepresivos, han experimentado también notables incrementos ${ }^{21-24}$.

Para la interpretación de los datos de la base ECOM utilizados para el presente estudio deben considerarse también algunas de sus limitaciones. Por un lado, la base de datos ECOM no incluye las prescripciones realizadas para los beneficiarios de ISFAS (Instituto Social de las Fuerzas Armadas), MUFACE (Mutualidad de Funcionarios Civiles del Estado) o MUGEJU (Mutualidad General Judicial), ni datos de otras mutualidades o de consumo directo con receta médica o de venta directa sin receta médica para especialidades que la requieren. Por otro lado, tampoco incluye el consumo en el medio hospitalario ni el de especialidades publicitarias o no incluidas en la prestación farmacéutica. Por lo tanto, los datos ofrecidos por esta base subestiman el uso real de antipsicóticos en los medios estudiados. Para evaluar la proporción de consumo 
extrahospitalario no incluido en ECOM se han utilizado como referencia los datos de los años 2000 y 2001 procedentes de la empresa IMS. En este caso también quedan excluidos los siguientes canales: ventas a hospitales o clínicas, botiquines de empresa y escolares, residencias de instituciones públicas o privadas que adquieran directamente la medicación, botiquines de las Fuerzas Armadas, o farmacias militares, etc.

Por otra parte, para el presente estudio se ha utilizado la DDD como unidad técnica de medida siguiendo las directrices de la OMS; aunque en el proceso de selección de la DDD para cada fármaco se tienen en cuenta las dosis recomendadas y, por tanto, las que presumiblemente se utilizan, a veces existen diferencias entre la DDD y la dosis realmente utilizada en la práctica clínica. En el caso de que las dosis realmente utilizadas sean superiores a la DDD, nuestros cálculos sobreestimarían el consumo real.

Asumiendo como consumo real el suministrado por IMS, se ha podido estimar que un $14 \%$ del consumo total de antipsicóticos en Castilla y León se realizó al margen del SNS, es decir, a través de recetas privadas, a través de mutuas o incluso mediante la obtención sin receta médica de medicamentos de prescripción. Este valor concuerda con el obtenido en otro estudio que ha analizado para otros medicamentos las diferencias entre ambas bases de datos ${ }^{25}$. Llama la atención, sin embargo, el que las diferencias sean ligeramente mayores para los antipsicóticos atípicos, de coste muy superior.

Entre las razones que explicarían las diferencias encontradas entre las distintas provincias, las desiguales prevalencias de enfermedad debidas a diferencias sociales (proporción de ancianos, por ejemplo) o a hábitos de prescripción parecen las más razonables $^{11}$. Estas diferencias entre provincias han sido destacadas para otros subgrupos de medicamentos psicotropos, lo que podrían indicar diferencias interprovinciales en la atención psiquiátrica (diferentes tipos de concertación para el suministro de medicamentos a determinadas plazas residenciales, por ejemplo) $)^{12,26}$. Estos mismos aspectos podrían explicar las diferencias encontradas para las distintas provincias entre ambas bases de datos. El hecho de que sea Segovia la provincia con menor consumo con cargo al SNS, y con mayores diferencias entre ambas bases de datos podría sugerir una mayor utilización privada. Con los datos utilizados no se puede saber si existe un patrón estacional de consumo; parece posible que los descensos observados en los meses de abril, julio y diciembre se deban a los periodos vacacionales.

Por último, el aumento explosivo del consumo de los antipsicóticos plantea abiertamente la adecuación de su utilización. Teniendo en cuenta que el incremento en los costes es aún más espectacular, en el supuesto de que existiera un pequeño porcentaje de casos en que el uso de estos fármacos fuera inadecuado éste representaría un montante económico considerable. Intervenciones administrativas a este respecto que encerrasen una propuesta formativa sobre el uso racional de estos medicamentos podrían optimizar los tratamientos con antipsicóticos en beneficio de los pacientes y de la sociedad en su conjunto.

\section{BIBLIOGRAFÍA}

1. Carvajal A, Martín Arias LH. Antipsychotics drugs. En: J.K: Aronson, ed. Side Effects of Drug Annual 25. Amsterdam: Elsevier Science BV 2002. p. 53-77.

2. Anónimo. Grupos terapéuticos y principios activos de mayor consumo en el Sistema Nacional de Salud durante 2001. Inf Ter Sist Nac Salud 2002; 26 (3): 78-83.

3. Uri A. Seguros de salud en España. El trebol digital-Revista de Mapfre. Nº11. Abril de 1999: 1-3. Disponible en: www.mapfrere.com/servicios/trebol_deta_es.asp?num=11\# Citado el: 15-01-2003.

4. Bases de datos de medicamentos de la Dirección General de Farmacia y Productos Sanitarios.

Rev Esp Salud Pública 2003, Vol. 77, N. ${ }^{\circ} 6$ 
Monografías Técnicas. Madrid: Ministerio de Sanidad y Consumo; 1989.

5. Instituto Nacional de Estadística. Disponible en: http://www.ine.es/tempus/cgi-bin/itie. Citado el 15-01-2003

6. WHO Collaborating Centre for Drug Statistics Methodology. Anatomical Therapeutic Chemical (ATC) classification index including Defined Daily (ATC) classification index including Defined Daily Doses (DDDs) for plain s
Oslo: WHO CCDSM; 2001.

7. Lunde PKM, Baksaas I, Halse M, et al. The methodology of drug utilization studies. In: Bergman $\mathrm{U}$, Grimsson A, Wahba AHW, Werterholm B (ed). Studies in drug utilization. Copenhague: WHO Regional Office for Europe; 1979. p. 17-28.

8. Instituto Nacional de Estadística. Disponible en: http://www.ine.es/daco/ipc.htm. Citado el: 15-012003.

9. Real Decreto $1663 / 1998$, de 24 de julio, por el que se amplía la relación de medicamentos a efectos de su financiación con cargo a fondos de la Seguridad Social o a fondos estatales afectos a la sanidad. BOE núm 177, de 25/07/1998.

10. Nordic Council on Medicines. Nordic Statistics on Medicines 1990-1992 (NLM Publication No 34 ). Uppsala: NLM; 1990.

11. Santamaría B, Perez M, Montero D, Madurga M, de Abajo FJ. Use of antipsychotic agents in Spain through 1985-2000. Eur Psychiatry 2002; 17(8): throug.

12. Ruiz Clavijo Díez MT, Sainz de Rozas Aparicio C Estudio del consumo de antipsicóticos en La Rioja. Años 1997-1998. impacto de los nuevos antipsicóticos. Pharm Care Esp 2000; 2: 338-46.

13. Dirección General de Farmacia y Productos Sanitarios. Circular $\mathrm{n}^{\circ}$. 10/93 sobre reglamentación específica para la prescripción, dispensación y utilización de leponex. Madrid: Ministerio de Sanidad y Consumo; 1993.

14. Kane JM. Schizophrenia. N Engl J Med 1996; 334(1): 34-41.

15. Killian JG, Kerr K, Lawrence C, Celermajer DS Myocarditis and cardiomyopathy associated with clozapine. Lancet 1999; 354(9193): 1841-5.
16. Committee for proprietary medicinal products opinion following an article 36 referral sertindole. London, 13 septiembre de 2002. EMEA/CPMP/ $2852 / 02$.

17. Vázquez-Barquero JL, Garcia J. Deinstitutionalization and psychiatric reform in Spain. Eur Arch Psychiatry Clin Neurosci 1999; 249(3): 128-35.

18. Love RC. Strategies for increasing treatment compliance: the role of long-acting antipsychotics. Am J Health Syst Pharm 2002; 59(22 Suppl 8): S10-5.

19. Glick ID, Murray SR, Vasudevan P, Marder SR, Hu RJ. Treatment with atypical antipsychotics: new indications and new populations. J Psychiatr Res 2001; 35(3): 187-91.

20. Consejo General de Colegios Oficiales de Farmacéuticos (CGCOF). Catálogo de Especialidades Farmacéuticas 2002. Madrid: Consejo General de Colegios Oficiales de Farmacéuticos; 2003.

21. Alonso MP, de Abajo FJ, Martínez JJ, Montero D, Martín-Serrano G, Madurga M. Evolución del consumo de antidepresivos en España. Impacto de los inhibidores selectivos de la recaptación de serotonina. Med Clin (Barc) 1997; 108(5): 161-6.

22. Rayon P, Montero D, Santamaria B, Madurga M, De Abajo FJ. Benzodiazepines consumption in Spain. Eur J Clin Pharmacol 1997; 52(4): 321-3.

23. Martin Arias LH, Carvajal A, Martin De Diego I, De Abajo F. Before and after triazolam: changes in the consumption pattern of hypnotics in Spain. Br J Clin Pharmacol 1995; 40(3): 289-90.

24. García del Pozo J, de Abajo FJ, Carvajal A, Montero D, Madurga M, García del Pozo V. Utilización de ansiolíticos e hipnóticos en España (19952001). Rev Clin Esp. En prensa.

25. De Las Cuevas C, Sanz EJ, Morán N, De La Fuente J. Benzodiazepines prescription is different in the public and private sectors. Pharmacoepidemiol Drug Saf 1999; 8: 351-3.

26. Gómez Juanes V, Candas Villar MA, Fidalgo González S, Armesto Gómez J, Calvo Alcántara MJ, de Marino Gómez-Sandoval MA, Vicens Caldentey C. Análisis del consumo de medicamentos utilizando indicadores de calidad en la prescripción. Aten Primaria 2000; 25(9): 618-24. 